\title{
Divertículo de Meckel volvulado asociado a brida como causa de obstrucción en intestino delgado
}

\author{
Juan Luis Morales G. ${ }^{1,2}$, Romina Olmos-de-Aguilera A. ${ }^{2,3}$, \\ Cristóbal Sánchez T. ${ }^{2,4}$ y Diego Sáez Q.,
}

'Servicio de Cirugía Hospital Clínico Herminda Martín, Chillán

Universidad Católica de la Santísima Concepción, Chillán. ${ }^{3}$ CESFAM Isidora Jiménez, Tirúa.

${ }^{4}$ CESFAM Violeta Parra, Chillán ${ }^{5}$ Hospital Huepil, Tucape

Recibido el 14 de junio de 2017 y aceptado para publicación el 2 de agosto de 2017.

Correspondencia a: Dra. Romina Olmos de Aguilera A.

raolmosdeaguilera@medicina. ucsc.cl

\section{Volvulated Meckel's diverticulum associated to intestinal adhesion as a cause of small bowel obstruction}

Introduction: Meckel's diverticulum is the most frequent gastrointestinal anomaly. It's more frequently seen in children less than 2 years of age. It's caused by the incomplete obliteration of the omphalomesenteric conduct. Case report: 19 year old male patient with no previous medical history presents sudden onset of high intensity abdominal pain, initially located in the periumbilical region, radiating to right lower quadrant associated with emesis, fever and tachycardia. Physical examination showed abdominal guarding, rebound tenderness and decreased bowel sounds. During McBurney laparotomy a large volume of viscous free fluid was found and signs of small bowel obstruction, deciding close McBurney laparotomy and performing sub-umbilical midline laparotomy. Meckel diverticulum is founded, twisted and strangled, with a bridle adhesion to the mesenteric root, compromising the small bowel. The adherence and the MD are resected, obtaining appropriate peristaltic activity and color. Appendectomy and peritoneal lavage are performed without complications. Discussion: preoperatory diagnosis of MD is unfrequented and its need surgical exploration. MD's volvulus is infrequent and in our case, intestinal obstruction it's secondary to an adherence between the volvulated MD and the mesentery.

Key words: abdomen acute; Meckel diverticulum; intestinal obstruction.

\section{Resumen}

Introducción: El divertículo de Meckel (DM) es una anomalía congénita gastrointestinal producto de obliteración incompleta del conducto onfalomesentérico, habitualmente en menores de dos años. Como complicación destaca obstrucción intestinal, intususcepción e inflamación. Caso Clínico: Paciente de sexo masculino, 19 años, sin antecedentes mórbidos. Ingresa a Urgencias por dolor abdominal, $24 \mathrm{~h}$ de evolución, que inicia en mesogastrio y luego irradia a fosa ilíaca derecha (FID), intenso, asociado a vómitos, fiebre y taquicardia. Al examen abdominal destaca resistencia muscular, dolor en FID, Blumberg positivo y ruidos hidroaéreos disminuidos. Se realiza laparotomía de McBurney hallándose gran cantidad de líquido libre citrino viscoso y signos de obstrucción de intestino delgado; decidiendo cierre de laparotomía de McBurney y realizar laparotomía media infraumbilical. Se encuentra divertículo de Meckel torcido y estrangulado, con brida hacia raíz del mesenterio de intestino delgado distal. Se realiza resección DM y bridolisis, recuperando vitalidad de asa y apendicectomía profiláctica. Discusión: El diagnóstico preoperatorio del DM es infrecuente y requiere exploración quirúrgica. El DM volvulado es raro, en nuestro caso produjo obstrucción intestinal secundaria a brida entre DM y mesenterio.

Palabras clave: abdomen agudo; divertículo de Meckel; obstrucción intestinal.

\section{Introducción}

El divertículo de Meckel es la anomalía gastrointestinal congénita más frecuente, corresponde a un divertículo verdadero compuesto por todas las capas del intestino delgado, que surge por la cara antimesentérica de la porción media o distal del íleon como resultado de la obliteración incompleta del conducto onfalomesentérico que normalmente debiera involucionar entre la $5^{\mathrm{a}}$ y $6^{\mathrm{a}}$ semana de gestación ${ }^{1,2}$. Tiene 
CASOS CLínicos

una incidencia global que va del 0,3 al $3 \%$, sin una predisposición familiar demostrada. La ocurrencia es igual tanto en hombres como en mujeres, pero la presentación de complicaciones es mayor en hombres. En general, a mayor edad disminuye riesgo de complicaciones, dentro de estas destaca la obstrucción intestinal secundaria a intususcepción o bridas correspondiente a más del $50 \%$; otras pueden ser ulceración, diverticulitis y perforación ${ }^{3,4}$.

Reportamos el caso de un paciente de 19 años en el que se realiza el diagnóstico intraoperatorio de obstrucción intestinal secundaria a un divertículo de Meckel volvulado asociado a una brida mesentérica.

\section{Presentación del caso}

Paciente de sexo masculino, 19 años, sin antecedentes mórbidos. Ingresa a Servicio de Urgencias por dolor abdominal de un día de evolución, que inicia en mesogastrio y luego se irradia a fosa ilíaca derecha (FID), de severa intensidad, asociado a varios episodios de vómitos, fiebre e hidratación límite, con ausencia de deposiciones y tránsito de gases conservado. Signos vitales de ingreso: frecuencia cardiaca $114 \mathrm{lpm}$, frecuencia respiratoria $20 \mathrm{rpm}$, presión arterial 115/82 $\mathrm{mmHg}$, temperatura axilar $38,7{ }^{\circ} \mathrm{C}$ y saturación de oxígeno $99 \%$ (con oxígeno ambiental). Al examen se constata abdomen poco depresible, resistencia muscular involuntaria, doloroso a palpación superficial y profunda en FID, signo de Blumberg positivo y ruidos hidroaéreos disminuidos. En exámenes de laboratorio destaca: hemoglobina $13 \mathrm{~g} / \mathrm{dl}$, hematocrito $40,5 \%$, leucocitos 15.300, plaquetas 390.000, PCR $35 \mathrm{mg} / \mathrm{dl}$. Se decide ingresar a pabellón con diagnóstico de abdomen agudo de origen apendicular.

Se realiza laparotomía de McBurney hallándose gran cantidad de líquido libre citrino viscoso, mucoideo, apéndice inflamado y signos de obstrucción de intestino delgado, decidiendo cierre laparotomía de McBurney y realizar laparotomía media infraumbilical exploradora. Se encuentra divertículo de Meckel torcido y estrangulado, con brida hacia raíz del mesenterio de intestino delgado distal, con obstrucción intestinal secundaria (Figura 1). Se realiza resección de DM y bridolisis (Figura 2), recuperando vitalidad de asa afectada y apendicectomía profiláctica, sin complicaciones.

Biopsia de pieza operatoria informa estructura tubular con fondo ciego que mide $6,5 \times 3,5 \times 2,5 \mathrm{~cm}$, revestida por serosa acentuadamente hemorrágica y lumen con abundante detritus celular (Figura 3). A nivel microscópico se describe divertículo verdadero

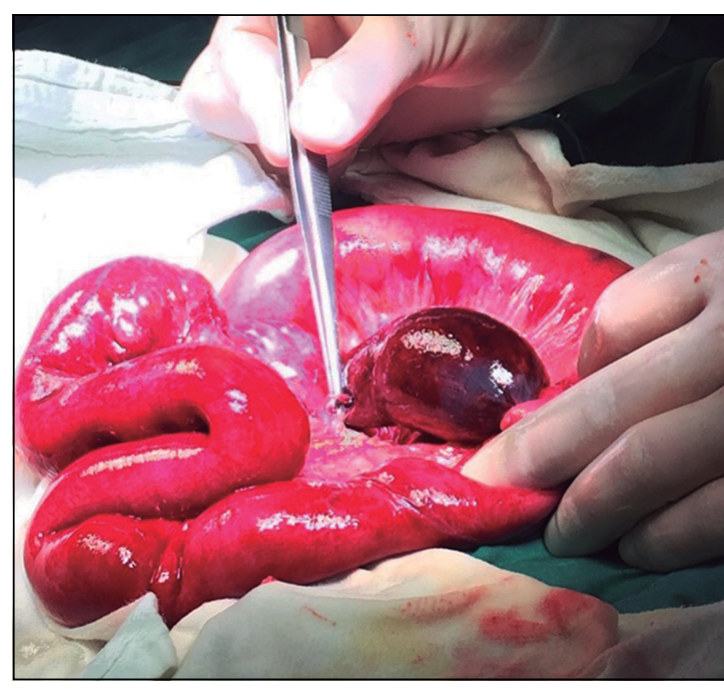

Figura 1. Divertículo de Meckel volvulado, necrótico y unido a mesenterio mediante brida.

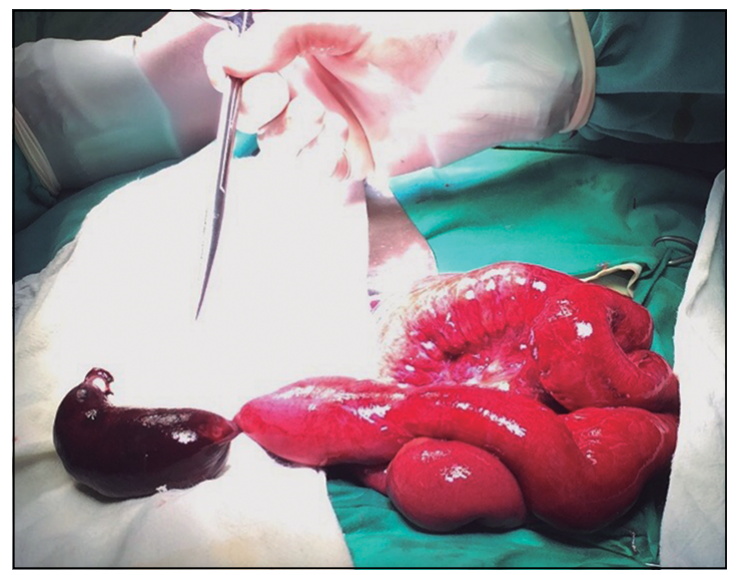

Figura 2. Divertículo de Meckel volvulado, parcialmente resecado. 


\section{CASOS CLÍNICOS}

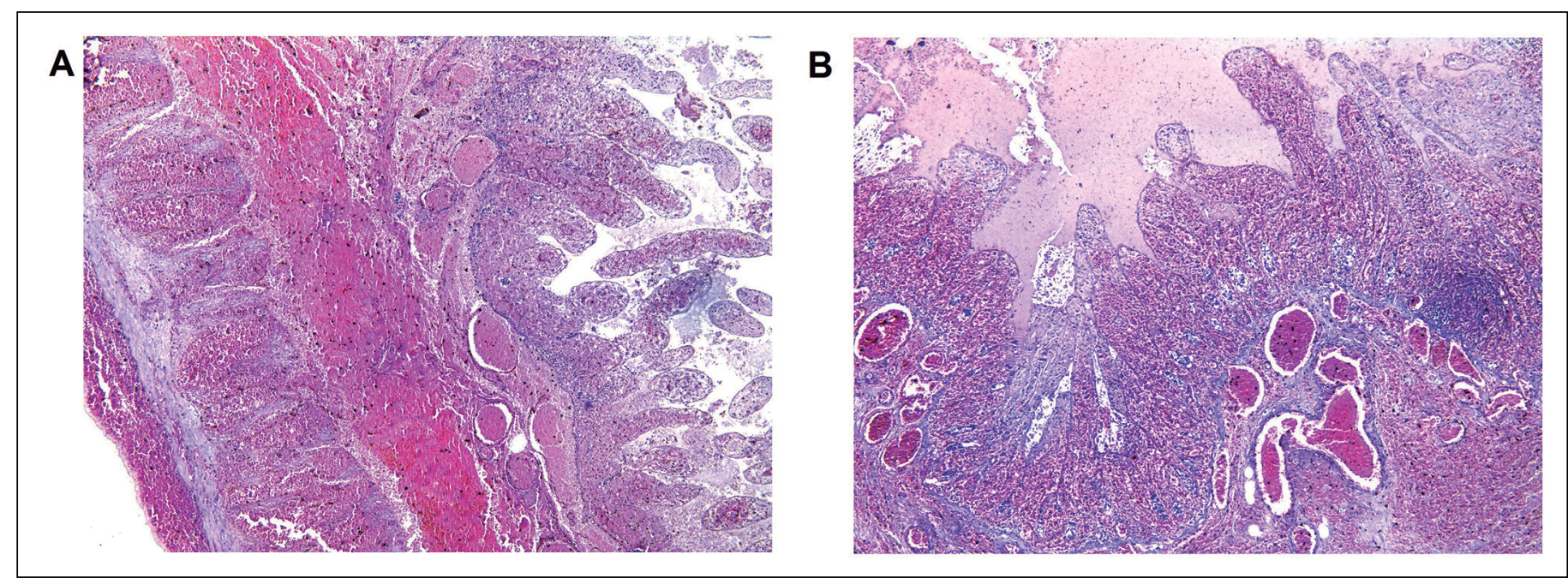

Figura 4. Biopsia pieza operatoria, tinción hematoxlina-eosina. (A) Divertículo verdadero conformado por mucosa, submucosa, túnica muscular y serosa (4x). (B) Mucosa y submucosa diverticular con vellosidades necróticas, infiltración hemorrágica y ectasia vascular (10x).

conformado por mucosa, submucosa, túnica muscular y serosa (Figura 4A), presenta vellosidades necróticas e infiltración hemorrágica, sin evidencia de mucosa ectópica (Figura 4B).

Paciente evolucionó favorablemente en el posoperatorio, se mantiene con tratamiento antibiótico biasociado (ceftriaxona metronidazol) por siete días y analgesia, presentando buena tolerancia a realimentación progresiva, con alta hospitalaria al séptimo día. En control posterior con cirujano en policlínico, a dos meses de la intervención, paciente en buen estado general, sin complicaciones.

\section{Discusión}

El conducto onfalomesentérico, que comunica saco vitelino e intestino medio durante la vida intrauterina, se oblitera y convierte en una banda fibrosa que conecta el ombligo con el intestino y lo provee de nutrición hasta que la placenta se termina de establecer. Todo esto normalmente entre la $5^{\mathrm{a}}$ y $9^{\mathrm{a}}$ semana de gestación ${ }^{2-4}$. La falta de involución parcial o completa de este conducto puede dar origen a varias estructuras potencialmente patológicas, de las cuales destacan: fístula onfalomesentérica, quiste onfalomesentérico, seno umbilical, bridas mesodiverticulares y divertículo de Meckel, que gana en frecuencia con un $98 \% \%^{5}$. El $90 \%$ de los divertículos se localizan hasta $90 \mathrm{~cm}$ de la válvula ileocecal, aunque algunos reportes han descrito divertículos hasta $180 \mathrm{~cm}$ de la válvula, y a pesar de que corresponde a tejido con todas las capas intestinales, el $50 \%$ de estos contienen tejido ectópico; de éstos, $60 \%-85 \%$ corresponden a tejido gástrico y $5 \%-16 \%$ a tejido pancreático ${ }^{6-8}$. En este caso, la biopsia informó pieza operatoria como divertículo verdadero, con las capas intestinales correspondientes, sin presencia de tejido ectópico.

La mayoría de los divertículos de Meckel se presentan como hallazgos de forma asintomática (33\%) dentro de un estudio por otra patología abdominal y en $16 \%$ como presentación de alguna complicación de este ${ }^{9}$. Estas complicaciones se manifiestan clínicamente como cuadros de abdomen agudo, hemorragia digestiva, obstrucción intestinal $\mathrm{o}$ inclusive peritonitis ${ }^{10}$. En el caso presentado, el dolor abdominal simuló un cuadro de apendicitis clásico, donde destaca la experiencia del cirujano para reconocer características inusuales del líquido intraabdominal y sospechar otra patología asociada.

En adultos las complicaciones más frecuentes del DM son la obstrucción asociada a adherencias o bridas o secundaria a intususcepción del divertículo hacia el íleon; sin embargo, algunos divertículos están adheridos al ombligo por una banda de tejido fibroso, lo que puede favorecer el desarrollo de una hernia interna o vólvulos ${ }^{11,12}$. Se postula que el mecanismo fisiopatológico es similar al de la diverticulitis colónica, con obstrucción del lumen que lleva a inflamación y eventualmente, puede condicionar perforación intestinal ${ }^{10}$. A pesar de las diversas presentaciones clínicas del divertículo de Meckel, la volvulación es una complicación rara, y mucho más infrecuente si presenta, además, brida 
a mesenterio. Las variaciones anatómicas que más favorecen la torsión de un divertículo de Meckel son una base estrecha y una importante longitud; ambas características estaban presentes en nuestro paciente ${ }^{13}$.

El abordaje quirúrgico del divertículo de Meckel depende del diagnóstico del mismo, si fue un hallazgo o si el paciente presentó sintomatología. Se deben considerar las complicaciones propias del divertículo y complicaciones secundarias al mismo, que repercuten en la integridad funcional y estructural de los tejidos adyacentes ${ }^{14}$. La resección ileal suele ser el tratamiento de elección, pues permite remover todo el tejido afectado y el ectópico; sin embargo, trabajos recientes muestran mayor tasa de morbilidad posoperatoria de resección intestinal frente a la diverticulectomía, por lo que se prefiere esta última ${ }^{14,15}$. En nuestro caso, a pesar del compromiso isquémico del DM e intestinal, no se evidenció la perforación del mismo y no fue necesario realizar resección ni anastomosis intestinal, pues se logró la exéresis indemne del DM y la recuperación satisfactoria de la vitalidad del intestino delgado en pocos minutos. El paciente evolucionó de manera favorable durante hospitalización, asintomático y sin complicaciones al control ambulatorio en cirugía.

\section{Responsabilidades éticas}

Protección de personas y animales. Los autores declaran que para esta investigación no se han realizado experimentos en seres humanos ni en animales.

Confidencialidad de los datos. Los autores declaran que en este artículo no aparecen datos de pacientes.

Derecho a la privacidad y consentimiento informado. Los autores declaran que en este artículo no aparecen datos de pacientes.

\section{Conflicto de intereses}

Los autores declaran no tener conflictos de interés.

\section{Agradecimientos}

Al Dr. Pedro Sandaña Jeno, Médico Anatomopatólogo del Hospital Clínico Herminda Martín de Chillán, por su excelente disposición y colaboración desde la especialidad para el análisis y presentación del caso clínico reportado.

\section{Bibliografía}

1. Passarge E, Stevenson RE. Meckel's diverticulum. In: Human malformations and related anomalies, $2^{\text {nd }}$, Stevenson RE, Hall JE (Eds), Oxford University Press, Oxford 2006. p.1111.

2. Mariño L, Fraga J, Rubio S, Segarra J, Gaetano M, Ossés JA. Persistencia del conducto onfalomesentérico. Arch Argent Pediatr. 2009;107:57-9.

3. Sagar J, Kumar V, Shah DK. Meckel's diverticulum: a systematic review. J R Soc Med. 2006;99:501-5.

4. Ruíz-Celorio M, Higuera-de la Tijera F, Pérez-Torres E. El divertículo de Meckel. Rev Med Hosp Gen Méx. 2014;77:88-92.

5. Chan SC, Lo CY. Meckel's diverticulum in action. N Engl J Med. 2004;350:e7.

6. Kim SW, Kim HC, Yang DM, Kim GY, Choi SI. MDCT findings of a Meckel's diverticulum with ectopic pancreatic tissue. Clin Imaging. 2014;38:70-2.

7. Tseng Y, Yang YJ. Clinical and diagnostic relevance of Meckel's diverticulum in children. Eur J Pediatr. 2009;168: 1519-23.

8. Burjonrappa S, Khaing P. Meckel's diverticulum and ectopic epithelium: Evaluation of a complex relationship. J Indian Assoc Pediatr Surg. 2014;19:859.

9. Park JJ, Wolff BG, Tollefson MK, Walsh EE, Larson DR. Meckel diverticulum: the Mayo Clinic experience with 1476 patients (1950-2002). Ann Surg. 2005;241:529-33.

10. Pepper VK, Stangill AB, Pearl RH. Diagnosis and management of pediatric appendicitis, intussusception, and Meckel diverticulum. Surg Clin North Am. 2012;92:505-26.
11. Beltrán $\mathrm{M}$, Larenas $\mathrm{R}, \mathrm{Almonacid} \mathrm{J}$, Danilova T, Cruces K, Barría C, et al. Vólvulo de divertículo de Meckel: una complicación inusual. Rev Chil Cir. 2006;58:50-3.

12. Estrella M, Bernal J, Fuenzalida C. Hernia interna de divertículo de Meckel como causa de obstrucción intestinal en un senescente. Rev Chil Cir. 2009;61:187-90.

13. Montenegro MA, Núñez $H$, Maroto L, Abril C, Gago P. Divertículo de Meckel torsionado con isquemia de asa intestinal. Cir Esp. 2009;85:54-63.

14. Yahchouchy EK, Marano AF, Etienne JC, Fingerhurt AL. Meckel's diverticulum. J Am Coll Surg. 2001;192:658-62.

15. Piñero A, Martínez E, Canteras M, Castellanos G, Rodríguez JM, Parrilla P. Complicaciones, diagnóstico y tratamiento del divertículo de Meckel. Cir Esp. 2001;70:286-90. 